\title{
lectures
}

\section{Construire la paix : conceptions collectives de son établissement, de son maintien et de sa consolidation}

par Séverine Autesserre

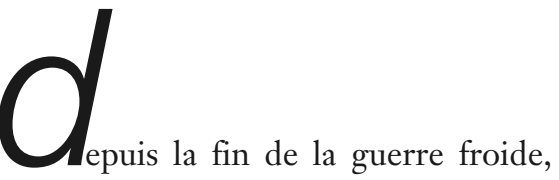
les interventions internationales destinées à rétablir la paix dans des zones en conflit se sont multipliées, tandis que les opérations des Nations unies, d'organisations non gouvernementales, de missions diplomatiques et d'organisations régionales croissaient en nombre et en envergure. Parallèlement, dans le champ des relations internationales, l'établissement de la paix (peacemaking), le maintien de la paix (peacekeeping) et la construction de la paix (peacebuilding) (notions ici réunies sous l'expression d'«interventions de paix $\gg$ ) ont suscité une riche littérature. Selon une acception large, la notion d'établissement de la paix désigne le processus par lequel des parties en conflit sont amenées à trouver un accord par des moyens pacifiques ; le maintien de la paix renvoie au déploiement de forces armées pour prévenir la reprise de violences à grande échelle après la signature d'un accord de paix ; enfin, la construction de la paix recouvre toutes les actions ayant vocation à renforcer et à consolider la paix ${ }^{1}$.

Dans les écrits de relations internationales, l'approche dominante - que l'on pourrait résumer sous le label de « théorie du choix rationnel »-met l'accent sur le rôle des intérêts et des contraintes matérielles dans la formulation des stratégies d'interventions de paix ${ }^{2}$. Ce courant de recherche a apporté une contribution importante à l'élucidation des conditions de l'engagement international, aux facteurs de succès ou d'échec des interventions et aux types

1. Sur la transformation de ces concepts, les visions contrastées de la paix qu'ils impliquent et la constitution du champ des études sur la paix (peace studies), voir Oliver Richmond, The Transformation of Peace, New York, Palgrave Macmillan, 2005. Sur la constitution et la transformation des recherches relatives au peacemaking, voir Jacques Faget, «Les métamorphoses du travail de paix. État des travaux sur la médiation dans les conflits politiques violents », Revue française de science politique, 58 (2), 2008, p. 309-333. Pour une approche de la construction de la paix « par le bas », voir Sandrine Lefranc, «Convertir le grand nombre à la paix... Une ingénierie internationale de pacification », Politix, 80 (4), 2007, p. 7-29.

2. Voir, par exemple, Michael Doyle, Nicholas Sambanis, Making War and Building Peace: United Nations Peace Operations, Princeton, Princeton University Press, 2006 ; Stephen John Stedman, Donald Rothchild, Elizabeth Cousens, Ending Civil Wars: The Implementation of Peace Agreements, Boulder, Lynne Rienner, 2002 ; William Zartman, Ripe for Resolution: Conflict and Intervention in Africa, New York, Oxford University Press, 1989. 
d'interventions les plus à même de réussir ${ }^{3}$. Néanmoins, cette approche paraît problématique à double titre. Premièrement, parce qu'elle n'interroge pas les processus de construction des intérêts comme des contraintes matérielles. Deuxièmement, parce qu'elle envisage les échecs comme un « problème auquel des solutions techniques peuvent être apportées » via, entre autres, l'allocation de ressources supplémentaires ou un engagement plus robuste ${ }^{4}$.

Se démarquant de cette grille de lecture dominante, une seconde approche est apparue récemment, qui prend pour principal objet les croyances, cultures, discours, cadres cognitifs (frames), habitus, identités, idéologies, normes, représentations, symboles et visions du monde (termes ici rassemblés sous la notion de « conceptions collectives » ou «partagées ») et étudie leurs incidences sur les interventions de paix. Bien qu'ils se revendiquent d'écoles théoriques variées, les auteurs mobilisant ces concepts sont souvent désignés dans le milieu des politistes sous l'appellation de « constructivistes » en raison de leur rejet de la théorie dominante du choix rationnel. Ils partagent en effet une vision commune de la causalité : si elles ne peuvent être tenues pour la «cause »d'une action spécifique, ni pour un facteur « déterminant», les interprétations collectives des enjeux rendent certaines décisions possibles et d'autres improbables ${ }^{5}$.

Certes, le nombre des études constructivistes consacrées aux interventions de paix reste limité au regard des travaux se réclamant du choix rationnel. Leur poids relatif et leur développement suggèrent toutefois l'utilité d'en proposer une cartographie. Le présent état des lieux souhaite en outre combler un déficit dans cette littérature, à savoir la rare recension et la faible mobilisation du large corpus des recherches anthropologiques (l'on concèdera que la réciproque est également vraie : la majorité des anthropologues ne mentionnent que rarement les écrits de relations internationales). Cette négligence s'explique probablement par la faible valorisation de l'interdisciplinarité dans la formation universitaire dispensée dans ces deux disciplines. Elle ne laisse pas moins de surprendre dans la mesure où anthropologues et constructivistes internationalistes se posent fondamentalement la même

\footnotetext{
3. Outre les ouvrages mentionnés en note 2, l'on pourra consulter Barbara Walter, Committing to Peace: The Successful Settlement of Civil Wars, Princeton, Princeton University Press, 2002 ; Virginia Page Fortna, Does Peacekeeping Work? Shaping Belligerents' Choices after Civil War, Princeton, Princeton University Press, 2008 ; Lise Morjé Howard, UN Peacekeeping in Civil Wars, New York, Cambridge University Press, 2008.

4. Robert Rubinstein, Peacekeeping under Fire: Culture and Intervention, Boulder, Paradigm Publishers, 2008, p. 9.

5. Voir James Fearon, Alexander Wendt, « Rationalism Versus Constructivism: A Skeptical View », dans Walter Carlsnaes, Thomas Risse, Beth Simmons (eds), Handbook of International Relations, Londres, Sage Publications, 2002, p. 58 ; Martha Finnemore, « Norms, Culture, and World Politics: Insights from Sociology's Institutionalism », International Organization, 50 (2), 1996, p. 325-347.
} 
question : comment l'intelligence collectivement partagée des situations influence-t-elle les interventions de paix? Il n'est d'ailleurs pas rare qu'anthropologues et constructivistes choisissent les mêmes cas d'études, par exemple les interventions en Somalie ou au Cambodge. De fait, la littérature anthropologique sur les rapports entre représentations collectives et interventions de paix est plus étoffée que celle de relations internationales car elle a émergé au milieu des années 1980, soit plus d'une décennie avant les premières recherches constructivistes sur ce sujet.

En raison de ces similitudes, on ne peut que souligner la complémentarité des deux littératures. Là où les internationalistes ont tendance à adopter une approche «par le haut » (top-down) et à centrer leur attention sur les décideurs aux sièges des organisations internationales et dans les capitales, les anthropologues privilégient souvent des analyses «par le bas » (bottom-up), qui explorent le déploiement sur le terrain d'interventions spécifiques. Comme le démontrent les rares exceptions à cette règle et les travaux de politique comparée, d'aires culturelles et d'anthropologie politique, seules des analyses combinant lectures «par le haut» et «par le bas », fondées sur une association entre les questionnements et les méthodes de science politique et d'anthropologie, sont en mesure de fournir une lecture nuancée et globale du rôle des conceptions collectives dans les interventions de paix ${ }^{6}$. Par-delà la rigidité des frontières disciplinaires, l'utilisation du concept de culture par les anthropologues explique peut-être la réticence des internationalistes à mobiliser leurs travaux. Nombreux sont les politistes qui jugent ce concept politiquement et idéologiquement connoté et considèrent qu'il ne saurait restituer la complexité du monde social ${ }^{7}$. Toutefois, à l'instar des politistes, les anthropologues rejettent désormais la conception autrefois dominante de la culture en tant que « régularités (patterns) homogènes et statiques

6. Voir, par exemple, David Ambrosetti, «Urgences et normalités de gestionnaires face aux violences "des autres". L'ONU et le Soudan », Actes de la recherche en sciences sociales, 174 (4), 2008, p. 81-99; Séverine Autesserre, « Hobbes and the Congo. Frames, Local Violence, and International Intervention », International Organization, 63, 2009, p. 249-280, et S. Autesserre, The Trouble with the Congo: Local Violence and the Failure of International Peacebuilding, New York, Cambridge University Press, 2010 ; Nathalie Duclos (dir.), L'adieu aux armes : parcours d'anciens combattants, Paris, Karthala, 2010 ; Didier Fassin, Mariella Pandolfi (eds), Contemporary States of Emergency: The Politics of Military and Humanitarian Interventions, New York, Zone Books, 2010 ; Vincent Foucher, « "Tradition africaine" et résolution des conflits. Un exemple sénégalais », Politix, 80 (4), 2007, p. 59-80; S. Lefranc, « Convertir le grand nombre à la paix... Une ingénierie internationale de pacification », art. cité ; Béatrice Pouligny, Ils nous avaient promis la paix : opérations de l'ONU et populations locales, Paris, Presses de Sciences Po, 2004 ; Roland Marchal, « The Roots of the Darfur Conflict and the Chadian Civil War», Public Culture, 20 (3), 2008, p. 429-436, et S. J. Stedman, Peacemaking in Civil War: International Mediation in Zimbabwe, 1974-1980, Boulder, Lynne Rienner, 1991. Voir aussi le dossier « Crises et organisations internationales », Cultures et Conflits, 75, automne 2009.

7. Sur cette question et sur les enjeux discutés dans le reste de ce paragraphe, voir R. Rubinstein, Peacekeeping under Fire: Culture and Intervention, op. cit., p. 41-48 et Kevin Avruch, Culture and Conflict Resolution, Washington, United States Institute of Peace Press, 1998, $1^{\text {ère }}$ partie. 
déterminant le comportement» et «uniformément distribuées parmi les membres d'un groupe $\gg^{8}$. Les anthropologues qui réfléchissent, comme Robert Rubinstein et Kevin Avruch, aux cadres d'interprétation des interventions de paix envisagent la culture comme « une activité (...) dynamique qui oriente et contraint (...) le comportement et autorise ainsi de considérables variations intraculturelles $\gg$, sans interdire pour autant l'existence de contradictions internes ${ }^{9}$. Une telle approche de la causalité n'est pas éloignée de celle défendue par les constructivistes internationalistes.

Plus généralement, anthropologues et constructivistes s'accordent à penser, avec les chercheurs en anthropologie politique et les spécialistes des aires culturelles, que les opérations de paix sont modelées et modulées par les mises en signification dont elles font l'objet. Intervenants et populations locales proviennent d'horizons nationaux, régionaux, professionnels, organisationnels, ethniques et religieux inscrits dans des univers culturels variés. Ces derniers influencent les théories des acteurs relatives aux causes de la paix et de la guerre; ils «fournissent le contexte dans lequel les croyances et les actions des agents sont construits, exprimés, interprétés et compris ${ }^{10}$. Il est toutefois important de noter que ces interprétations, prépondérantes dans un contexte culturel donné, ne sont pas partagées au même degré par tous les acteurs internationaux : les identités et les sous-cultures, la définition des intérêts et des contraintes varient d'une organisation à l'autre comme en leur sein. Ces institutions internationales proposent également des visions contrastées de la paix et des interventions.

La plupart des chercheurs pensent en outre - deuxième convergence entre disciplines - que les cadres d'intelligibilité influencent les interventions plus profondément que les contextes politiques, économiques et sociaux ou les intérêts établis et les contraintes matérielles. De leur point de vue, les cadres culturels, normatifs, professionnels et idéologiques des acteurs, tout comme leurs perceptions du temps et de l'histoire, façonnent les interprétations des contraintes, intérêts et contextes. Enfin, un certain nombre d'internationalistes constructivistes et d'anthropologues souhaitent valoriser les implications décisionnelles de leur recherche. Ils pensent pouvoir éclairer l'issue des interventions et, ce faisant, contribuer à accroître leur efficacité ${ }^{11}$.

8. K. Avruch, Culture and Conflict Resolution, op. cit., p. 14. Toutes les citations ont été traduites par nos soins.

9. R. Rubinstein, Peacekeeping under Fire: Culture and Intervention, op. cit., p. 42.

10. Tamara Duffey, «Cultural Issues in Contemporary Peacekeeping », International Peacekeeping, 7 (1), 2000, p. 142-168, citation p. 143 ; voir également Betts Fetherston, Carolyn Nordstrom, « Overcoming Habitus in Conflict Management: UN Peacekeeping and Warzone Ethnography », Peace and Change, 20 (1), 1995, p. 94.

11. S. Autesserre, The Trouble with the Congo: Local Violence and the Failure of International Peacebuilding, op. cit.; T. Duffey, « Cultural Issues in Contemporary Peacekeeping », art. cité ; O. Richmond, The Transformation of Peace, op. cit. ; R. Rubinstein, Peacekeeping under Fire: Culture and Intervention, op. cit.. 
Le présent état de littérature se propose dès lors d'explorer conjointement les apports des littératures anthropologique et constructiviste, les débats qui les traversent, leurs complémentarités et leurs conflits, et de mettre en lumière leurs limites. Dans un premier temps, l'analyse abordera les approches « par le haut », structurées autour de deux thèmes principaux : la culture diplomatique et les styles de négociation nationaux, d'une part; le paradigme de la paix libérale, d'autre part. Après avoir mis en évidence les apories de ces approches, l'on explorera deux controverses clés des approches «par le bas»dans les écrits constructivistes : l'existence (ou non) de divergences entre les répertoires culturels des intervenants et ceux des populations locales, d'abord, l'enjeu des cadres professionnels et organisationnels des entrepreneurs de paix, ensuite. En conclusion, plusieurs pistes de recherche seront suggérées.

\section{Les approches " par le haut ": les décideurs aux sièges des organisations internationales et dans les capitales}

\section{Culture diplomatique et styles de négociation}

Les chercheurs qui s'intéressent aux interventions de paix mobilisent une vaste littérature qui s'attache à décrire l'influence des cultures professionnelles nationales sur les pratiques de négociation ${ }^{12}$. Née dans la seconde moitié du XXe siècle, cette littérature se concentre habituellement sur l'étude des diplomates de haut rang et des représentants de l'État. Deux approches peuvent y être distinguées. La première souligne l'existence de contrastes entre les styles de négociation privilégiés aux échelles nationale et régionale ${ }^{13}$. Elle défend l'idée selon laquelle les valeurs partagées à chacun de ces niveaux peuvent conditionner « la propension d'un gouvernement à engager une négociation, à recourir à des procédures d'adjudication ou d'arbitrage, ou encore à ignorer un enjeu ». Les cultures de négociation exerceraient également une influence sur la définition «du rythme ou du calendrier approprié à certaines pratiques de négociation, (...) de ce qui relève d'une obligation de

12. Voir les très précieux états de littérature proposés dans K. Avruch, Culture and Conflict Resolution, op. cit. (deuxième partie), et K. Avruch, « Culture as Context, Culture as Communication: Considerations for Humanitarian Negotiators », Harvard Negotiation Law Review, 9, 2004, p. 405 ; Michael Fowler, « Culture and Negotiations: The Pedagogical Dispute Regarding Cross-Cultural Negotiations », International Studies Perspective, 10, 2009, p. 341-359. L'ouvrage de Guy Olivier Faure et Jeffrey Rubin, Culture and Negotiations (Londres, Sage publications, 1993), est représentatif de cette littérature.

13. Voir, entre autres, Raymond Cohen, Culture and Conflict in Egyptian-Israeli Relations: A Dialogue of the Deaf, Bloomington, Indiana University Press, 1990 ; Edward Hall, Beyond Culture, Garden City, Anchor Press, 1976. 
réciprocité, (...) d'un résultat jugé équitable et juste (...) et de la conduite des négociations futures ${ }^{14}$. La seconde approche considère, a contrario, qu'il existe des similarités entre milieux diplomatiques. Indépendamment de leur pays ou région d'origine, les négociateurs partageraient une culture diplomatique internationale ${ }^{15}$ enracinée dans l'histoire et les valeurs occidentales. Cette caractéristique sert de pont avec la seconde controverse traversant les recherches « par le haut », à savoir le débat sur la paix libérale.

\section{Le débat sur la « paix libérale »}

Le paradigme de la paix libérale occupe depuis la fin des années 1990 une place croissante dans les travaux consacrés aux interventions de paix. Or, là où les tenants du choix rationnel se donnent pour objectif de déterminer dans quelle mesure les démocraties libérales sont capables d'assurer la paix chez elles comme sur la scène internationale ${ }^{16}$, les constructivistes, eux, interrogent la nature et l'influence de «l'agenda libéral pour la paix ». Dans la littérature constructiviste, le récit dominant souligne les similarités entre diplomates et fonctionnaires internationaux d'institutions aussi différentes que l'Union africaine, le Fonds monétaire international, les Nations unies, la Banque mondiale, les États-Unis ou diverses ONG. Ces intervenants partageraient une même culture mondiale (world-polity culture) adossée à des valeurs libérales occidentales, dont la prépondérance dans les arènes internationales favoriserait la convergence des stratégies et la défense d'un agenda libéral pour la paix comprenant la tenue d'élections, l'instauration d'une économie de marché et la promotion des droits de l'homme et de l'État de droit ${ }^{17}$.

En règle générale, les constructivistes dénoncent un agenda qu'ils jugent hégémonique et tiennent pour inadapté aux réalités des environnements postconflit $^{18}$. Plusieurs chercheurs ont également dénoncé la tendance des

\footnotetext{
14. M. Fowler, «Culture and Negotiations: The Pedagogical Dispute Regarding Cross-Cultural Negotiations », art. cité, p. 345 .

15. Voir William Zartman, Maureen Berman, The Practical Negotiator, New Haven, Yale University Press, 1982 ; Iver Neumann, « To Be a Diplomat », International Studies Perspectives, 6, 2005, p. 72-93. Sur la culture diplomatique, voir le classique James Der Derian, On Diplomacy: A Genealogy of Western Estrangement, Oxford, Blackwell, 1987.

16. Voir M. Doyle, « Kant, Liberal Legacies, and Foreign Affairs », Philosophy and Public Affairs, 12 (3), 1983, p. $205-$ 235 ; Zeev Maoz, Bruce Russett, « Normative and Structural Causes of the Democratic Peace, 1946-1986 », American Political Science Review, 87 (3), 1993, p. 624-638.

17. Pour une excellente présentation de cette thèse, voir Roland Paris, At War's End: Building Peace after Civil Conflict, Cambridge, Cambridge University Press, 2004.

18. Voir, entre autres, David Chandler, «Introduction: Peace without Politics », International Peacekeeping, 12 (3), 2005, p. 307-321 ; Mark Duffield, Global Governance and the New Wars: The Merging of Development and Security, New York, Zed Books, 2001 ; O. Richmond, The Transformation of Peace, op. cit., ainsi que les numéros spéciaux que la revue International Peacekeeping a consacrés à ce débat en $2004\left(n^{\circ} 1\right)$ et en $2009\left(n^{\circ} 5\right)$.
} 
décideurs à offrir une lecture techniciste de la gestion et des sorties de conflits. Adoptant une approche énumérative (avec liste de critères à remplir), les intervenants supposeraient que chaque situation se prête à - et requiert - l'utilisation d'une boîte à outils qui inclut le déploiement de soldats de la paix, le désarmement, la démobilisation et la réintégration des combattants, le retour des réfugiés, la libéralisation de l'économie et l'organisation de scrutins électoraux ${ }^{19}$. Du point de vue des auteurs constructivistes, une telle démarche ne saurait apporter ni une paix durable ni une démocratie effective.

Leur argumentation a fait l'objet de deux contestations. La première est venue de théoriciens post-structuralistes pour qui les interventions de paix mettent en jeu des processus aux logiques plus subtiles que l'image d'une imposition pure et simple de valeurs occidentales ne le donnerait à penser. À leurs yeux, les opérations de paix contemporaines gagneraient à être envisagées comme une forme de biopolitique. Elles représentent un nouveau type de régulation internationale qui soustrait les détenteurs du pouvoir à l'obligation de rendre compte de leurs actions et interdit, ce faisant, le développement d'une démocratie ouverte ${ }^{20}$. La seconde critique a été formulée par des spécialistes de sociologie des organisations, sensibles à la diversité des intervenants. Mettant en avant l'importance des cadres organisationnels (organizational frames), ces chercheurs ont défendu l'idée selon laquelle chaque institution serait porteuse de conceptions propres du maintien et de la fabrique de la paix. Dans leur perspective, les cadres organisationnels sont avant tout déterminés par les cultures internes des bureaucraties internationales ${ }^{21}$. Le paradigme de la paix libérale ne constitue alors que l'un des schémas interprétatifs possibles influençant les actions des organisations internationales, au siège comme sur le terrain.

19. D. Chandler, «Introduction: Peace without Politics», art. cité, p. 308 ; B. Pouligny, Ils nous avaient promis la paix : opérations de l'ONU et populations locales, op. cit., notamment p. 293. Sur la création et la transformation de cette boîte à outils, voir Marina Ottaway, « Promoting Democracy after Conflict: The Difficult Choices », International Studies Perspectives, 4, 2003, p. 314-322.

20. D. Chandler, Empire in Denial: The Politics of State-Building, Londres, Pluto, 2006 ; François Debrix, Re-Envisioning Peacekeeping: The United Nations and the Mobilization of Ideology, Minneapolis, University of Minnesota Press, 1999; M. Duffield, Development, Security and Unending War: Governing the World of Peoples, Cambridge, Polity Press, 2007.

21. Michael Barnett, Eyewitness to a Genocide: The United Nations and Rwanda, Ithaca, Cornell University Press, 2002 ; M. Barnett, M. Finnemore, Rules for the World: International Organizations in Global Politics, Ithaca, Cornell University Press, 2004, et Susanna Campbell, « Organizational Barriers to Peace », thèse de doctorat, Tufts University, soutenance prévue en 2011. 


\section{Une vision partielle reposant sur des postulats erronés}

Si elle démontre de façon convaincante qu'une culture mondiale occidentale et libérale contribue, au même titre que les cadres régionaux, nationaux, organisationnels et professionnels, à conférer leurs formes singulières aux interventions de paix, la littérature consacrée à la paix libérale et aux négociations internationales n'en fournit toutefois qu'une vision parcellaire. Elle néglige souvent les pratiques concrètes et le quotidien de l'action internationale, les tensions sociales et épistémologiques entre acteurs internationaux et l'impact, sur les missions, de l'opinion publique et des considérations de politique intérieure ${ }^{22}$. Elle ignore également régulièrement qu'une connaissance approfondie d'études de cas spécifiques représente une condition nécessaire à toute élaboration théorique. Enfin, cette littérature n'accorde qu'une attention furtive à la manière dont les interventions internationales opèrent sur le terrain, c'est-à-dire dans les univers ruraux et les provinces où la plupart des pacificateurs sont déployés.

Les approches «par le haut» partent du postulat implicite que le niveau microsocial ne constituerait qu'une simple réplique du niveau macro, de sorte que les transformations enregistrées sur les scènes nationale et internationale ou les actions entreprises dans les hautes sphères politiques susciteraient automatiquement des transformations similaires au niveau local. De fait, les dynamiques locales de guerre et de paix diffèrent généralement de celles observables à l'échelle des États ${ }^{23}$. De même, les instructions émises depuis les capitales et les quartiers généraux des organisations internationales ne se traduisent pas mécaniquement par l'adoption de mesures concordantes sur le terrain, car les ordres doivent être interprétés. De plus, sur les sites décentralisés où ils interviennent, les membres des missions internationales disposent d'une marge de manœuvre significative dans la mise en œuvre des injonctions qu'ils reçoivent ${ }^{24}$. Enfin, comme nous allons le démontrer maintenant,

22. Pour de plus amples développements sur cette critique, voir David Ambrosetti, Yves Buchet de Neuilly, «Éditorial : les organisations internationales au cœur des crises. Configurations empiriques et jeux d'acteurs », Cultures et Conflits, 75, 2009, p. 7-14. Pour dépasser ces postulats, voir D. Ambrosetti, Normes et rivalités diplomatiques à l'ONU. Le Conseil de sécurité en audience, Bruxelles, PIE Peter Lang, 2009 ; Brent Steele, « Making Words Matter: The Asian Tsunami, Darfur, and "Reflexive Discourse" in International Politics », International Studies Quarterly, 51 (4), 2007, et le dossier de Cultures et Conflits sur « Crises et organisations internationales », cité.

23. S. Autesserre, The Trouble with the Congo: Local Violence and the Failure of International Peacebuilding, op. cit.; Stathis N. Kalyvas, The Logic of Violence in Civil War, New York, Cambridge University Press, 2006 ; Scott Straus, The Order of Genocide: Race, Power, and War in Rwanda, Ithaca, Cornell University Press, 2006.

24. Victoria Holt, Tobias Berkman, The Impossible Mandate? Military Preparedness, the Responsibility to Protect and Modern Peace Operations, Washington, The Henry L. Stimson Center, 2006 ; B. Pouligny, Ils nous avaient promis la paix : opérations de l'ONU et populations locales, op. cit., p. 159-164. 
les interprétations collectives des situations diffèrent en fonction de l'échelle considérée.

Les approches « par le bas » : les pacificateurs sur le terrain

\section{Interventions internationales et cultures locales}

L'essentiel des travaux de l'approche « par le bas » consacrés aux conceptions collectives de la paix et des interventions porte sur les cultures des populations locales et, plus particulièrement, sur les différences qu'elles présenteraient par rapport à celles des pacificateurs internationaux. Maints anthropologues et psychologues ont ainsi cherché à explorer les visions supposées singulières de la paix et de sa construction dans les sociétés qu'ils ont étudiées ${ }^{25}$. En outre, de nombreux chercheurs - et ce point est plus important pour notre sujet - ont identifié l'existence d'un contraste entre les conceptions de la paix, de son établissement et de son maintien défendues par les membres de sociétés locales (ainsi, par exemple, au Timor ou en Somalie) et les intervenants extérieurs ${ }^{26}$. Enfin, la plupart des textes font référence, ne serait-ce qu'implicitement, au débat sur la paix libérale, en rappelant que les acteurs internationaux utilisent des techniques de gestion des conflits inspirées par l'agenda libéral pour la paix et peu pertinentes au regard des sites sur lesquels ils interviennent.

L'argument central développé dans ces écrits réside dans l'affirmation qu'un déficit de « compétence culturelle »des intervenants se situerait au fondement des échecs des opérations de pacification. Ce déficit susciterait l'adoption de stratégies contreproductives, nuirait durablement à la popularité des intervenants et pourrait même engendrer des conflits entre acteurs internationaux et populations locales et/ou groupes

\footnotetext{
25. Voir l'analyse des travaux d'anthropologie sociale proposée dans Roger Mac Ginty, « Indigenous Peace-Making Versus the Liberal Peace », Cooperation and Conflict, 43 (139), 2008, p. 147-149. En psychologie, voir, par exemple, Luciana Karine de Souza et al., « Brazilian Children's Conceptions of Peace, War, and Violence », Peace and Conflict: Fournal of Peace Psychology, 12 (1), 2006, p. 49-63 ; Karen Myers-Bowman, Kathleen Walker, Judith Myers-Walls, « "Differences between War and Peace Are Big": Children from Yugoslavia and the United States Describe Peace and War », Peace and Conflict: Fournal of Peace Psychology, 11 (2), 2005, p. 177-198.

26. Voir, entre autres, Jarat Chopra, Tanja Hohe, «Participatory Intervention », Global Governance, 10, 2004, p. 289-305; T. Duffey, «Cultural Issues in Contemporary Peacekeeping», art. cité ; T. Hohe, «Clash of Paradigms: International Administration and Local Political Legitimacy in East Timor», Contemporary Southeast Asia, 24 (3), 2002, p. 569-589 ; B. Pouligny, Ils nous avaient promis la paix : opérations de l'ONU et populations locales, op. cit., en particulier p. 133-136; Paul Salem, « A Critique of Western Conflict Resolution from a Non-Western Perspective », dans P. Salem (ed.), Conflict Resolution in the Arab World: Selected Essays, Beirut, American University of Beirut, 1997.
} 
armés ${ }^{27}$. Selon les tenants de ce courant, les intervenants devraient essayer de s'ouvrir aux cultures locales et de mieux les connaître s'ils souhaitent accroître leurs chances de succès ${ }^{28}$. Cette découverte a donné naissance à une vaste littérature destinée aux praticiens qui y trouvent des conseils en matière de résolution interculturelle des conflits ${ }^{29}$.

\section{Les cultures organisationnelles et professionnelles}

Seules quelques recherches sur les interventions de paix ont tenté de dépasser une définition en termes d'Occident et de libéralisme des univers de sens des pacificateurs et ont entrepris d'explorer plus avant la diversité de leurs grilles de lecture. La plupart de ces études sont le fait d'anthropologues, plus rarement de psychologues. Elles prennent généralement pour objet les cultures organisationnelles et accordent une attention privilégiée aux casques bleus des Nations unies.

Par-delà l'analyse des différences culturelles entre acteurs internationaux et locaux, leurs réflexions s'ordonnent autour de quatre thèmes. Le premier concerne l'analyse des canaux à travers lesquels cultures nationales, organisationnelles et professionnelles influencent le choix des stratégies de construction de la paix ${ }^{30}$. Le second porte sur les tensions et les malentendus culturels entre les divers contingents des Nations unies et sur leur impact en matière

27. En dehors des sources citées en note 26, voir B. Fetherston, C. Nordstrom, « Overcoming Habitus in Conflict Management: UN Peacekeeping and Warzone Ethnography » art. cité ; Marianne Heiberg, « Peacekeepers and Local Populations: Some Comments on UNIFIL », dans Indar Jit Rikye, Kjell Skjelsbaek (eds), The United Nations and Peacekeeping, Basingstoke, Macmillan, 1990 ; R. Rubinstein, Peacekeeping under Fire: Culture and Intervention, op. cit., chapitre 7, et Donna Winslow, The Canadian Airborne Regiment in Somalia. A Socio-Cultural Inquiry, Ottawa, Commission of Inquiry into the Deployment of Canadian Forces to Somalia, 1997.

28. La littérature universitaire a essentiellement étudié les échecs. Parmi les rares exceptions, voir S. Campbell, « Organizational Barriers to Peace », cité, et R. Mac Ginty, « Indigenous Peace-Making Versus the Liberal Peace », art. cité, p. 152-155.

29. Voir, par exemple, Dan Landis, Rabi Bhagat, Handbook of Intercultural Training, Thousand Oaks, Sage Publications, 1996 ; Michelle LeBaron, Venashri Pillay, Conflict across Cultures: A Unique Experience of Bridging Differences, Boston, Intercultural Press, 2006, et John Paul Lederach, Preparing for Peace: Conflict Transformation across Cultures, Syracuse, Syracuse University Press, 1995. Voir également le débat suscité au sein de l'armée américaine par l'utilisation d'« équipes humaines de terrain » (Human Terrain Teams, HTT), équipes d'anthropologues déployées au sein de formations militaires, en Afghanistan notamment, afin d'améliorer la lutte contre les rébellions à travers une meilleure compréhension des contextes locaux.

30. B. Fetherston, C. Nordstrom, « Overcoming Habitus in Conflict Management: UN Peacekeeping and Warzone Ethnography » art. cité ; Robert Rubinstein, Diana Keller, Michael Scherger, « Culture and Interoperability in Integrated Missions », International Peacekeeping, 15 (4), 2008, p. 540-556 ; R. Rubinstein, Peacekeeping under Fire: Culture and Intervention, op. cit. ; D. Winslow, « Misplaced Loyalties: The Role of Military Culture in the Breakdown of Discipline during Peace Operations », Canadian Review of Sociology and Anthropo$\log y, 35$ (3), 1998, p. 345-367. 
d'efficacité des missions ${ }^{31}$. Ces travaux sont, eux aussi, traversés par le clivage entre une approche qui privilégie les cultures militaires nationales et des recherches qui les voient se fondre en une culture commune de maintien de la paix atténuant les divergences nationales ${ }^{32}$. Un troisième axe de réflexion concerne l'influence du genre sur les pratiques de maintien de la paix, notamment à travers la création, au sein des missions, d'une culture militariste masculine ${ }^{33}$. Enfin, certains écrits interrogent «la manière dont la culture contribue à faire du maintien de la paix une institution sociale » et à lui conférer une légitimité ${ }^{34}$.

Les études consacrées aux autres protagonistes (diplomates, personnel civil des missions de maintien de la paix, membres d'ONG) sont sensiblement plus rares et prioritairement ciblées sur les acteurs de l'humanitaire ou de l'aide au développement. Leur culture professionnelle spécifique conduirait les représentants de ces deux milieux à privilégier des stratégies techniques et apolitiques ${ }^{35}$. Ces cadres professionnels - et c'est le point le plus important pour notre propos - dissuaderaient les acteurs de l'aide de prendre activement part aux interventions de paix.

31. Eyal Ben-Ari, Efrat Elron, « Blue Helmets and White Armor: Multi-Nationalism and Multi-Culturalism among UN Peacekeeping Forces », City \& Society, 13 (2), 2001, p. 271-302 ; T. Duffey, « Cultural Issues in Contemporary Peacekeeping », art. cité ; M. Heiberg, « Peacekeepers and Local Populations: Some Comments on UNIFIL », cité ; Maren Tomforde, «Motivation and Self-Image among German Peacekeepers », International Peacekeeping, 12 (4), 2005, p. 576-585.

32. B. Fetherston, C. Nordstrom, « Overcoming Habitus in Conflict Management: UN Peacekeeping and Warzone Ethnography » art. cité ; R. Rubinstein, Peacekeeping under Fire: Culture and Intervention, op. cit. ; Donna Winslow, Peer Everts, «It's Not a Question of Muscle: Cultural Interoperability for NATO », dans Gustav Schmidt (ed.), A History of NATO - The First Fifty Years, Hampshire, Palgrave Macmillan, 2001.

33. Gurchathen Sanghera, Marsha Henry, Paul Higate, Peacekeepers as New Men? Security and Masculinity in the United Nations Mission in Liberia, Bristol, Centre for Governance and International Affairs, University of Bristol, 2008 ; Liora Sion, « Peacekeeping and the Gender Regime. Dutch Female Peacekeepers in Bosnia and Kosovo », Journal of Contemporary Ethnography, 37 (5), 2008, p. 561-585 ; Sandra Whitworth, Men, Militarism and UN Peacekeeping: A Gendered Analysis, Boulder, Lynne Rienner, 2004. Sur les dimensions raciales et genrées du maintien de la paix, voir aussi Sherene Razack, Dark Threats and White Knights: The Somalia Affair, Peacekeeping and the New Imperialism, Toronto, University of Toronto Press, 2004.

34. R. Rubinstein, Peacekeeping under Fire: Culture and Intervention, op. cit., p. 41. D'autres exemples peuvent être trouvés dans E. Ben-Ari, E. Elron, « Blue Helmets and White Armor: Multi-Nationalism and Multi-Culturalism among UN Peacekeeping Forces », art. cité ; Marianne Heiberg, Johan Jørgen Holst, « Peacekeeping in Lebanon: Comparing UNIFIL and the MNF », Survival, 28 (5), 1986, p. 399-422 ; L. Sion, «"Too Sweet and Innocent for War"? Dutch Peacekeepers and the Use of Violence », Armed Forces \& Security, 32 (3), 2006, p. 454-474.

35. Voir, parmi de nombreux autres travaux, D. Fassin, M. Pandolfi, Contemporary States of Emergency: The Politics of Military and Humanitarian Interventions, op. cit. ; James Ferguson, The Anti-Politics Machine: « Development », Depoliticization, and Bureaucratic Power in Lesotho, New York, Cambridge University Press, 1990 ; Peter Uvin, Aiding Violence: The Development Enterprise in Rwanda, West Hartford, Kumarian Press, 1998. Pour une étude approfondie du rôle de l'aide humanitaire et de l'aide au développement dans la sécurité internationale, voir M. Duffield, Global Governance and the New Wars: The Merging of Development and Security, op. cit.. 
Un autre corpus de travaux corrélé s'est penché sur les interactions entre milieux militaires et humanitaires (qu'il s'agisse d'ONG ou de branches civiles des missions des Nations unies). La plupart des auteurs s'accordent à penser que ces deux groupes sont porteurs d'une culture professionnelle propre. Leurs principales divergences résideraient dans les interprétations des notions de sécurité et de coordination. Les militaires, bien dotés en ressources matérielles et humaines, partageraient une culture de la hiérarchie et du contrôle social étroit, alors que les ONG tendraient à opérer de manière décentralisée, indépendante, et avec un personnel modeste ${ }^{36}$. Ces différences seraient à l'origine de tensions entre les acteurs civils et militaires de l'établissement de la paix, qui affecteraient la qualité de leur coopération et l'efficacité des missions internationales. Ces études se donnent dès lors pour finalité de contribuer à améliorer la coopération civilomilitaire en suggérant des mécanismes de coordination ou en favorisant une meilleure compréhension mutuelle.

\section{Trois pistes de recherche}

Cette littérature est convaincante lorsqu'elle entreprend de démontrer le rôle des cadres ou des cultures nationales, organisationnelles, professionnelles et du genre dans la mise en œuvre concrète des opérations de pacification sur le terrain. Elle fournit également plusieurs variables explicatives importantes des échecs des interventions, comme le manque d'ouverture sur les cultures locales et les divergences entre contingents nationaux ou entre civils et militaires. Cependant, en prenant pour objet d'étude presque exclusif les soldats de la paix, elle tend à reléguer au second plan trois enjeux ${ }^{37}$.

Premièrement, si elle fournit une bonne saisie des cadres d'interprétation mobilisés par les militaires, la littérature sur les opérations de paix « par le bas » n'offre aucune connaissance équivalente des autres univers professionnels (diplomates, civils, personnel des ONG actives dans le

\footnotetext{
36. D. Winslow, « Strange Bedfellows: NGOs and the Military in Humanitarian Crises », International fournal of Peace Studies, 7 (2), 2002, p. 113-129 ; R. Rubinstein, D. Keller, M. Scherger, « Culture and Interoperability in Integrated Missions », art. cité, p. 543 ; R. Rubinstein, Peacekeeping under Fire: Culture and Intervention, op. cit., chapitre 7. Voir aussi T. Duffey, «Cultural Issues in Contemporary Peacekeeping », art. cité ; Hugo Slim, « The Stretcher and the Drum: Civil-Military Relations in Peace Support Operations », International Peacekeeping, 3 (2), 1996, p. 123-140.

37. Des incursions dans ces domaines ont toutefois été menées avec succès. Voir, par exemple, S. Autesserre, The Trouble with the Congo: Local Violence and the Failure of International Peacebuilding, op. cit.; S. Campbell, «Organizational Barriers to Peace », cité ; S. Lefranc, « Du droit à la paix. La circulation des techniques internationales de pacification par le bas », Actes de la recherche en sciences sociales, 174 (4), 2008, p. 48-67 ; Carrie Manning, Monica Malbrough, « Bilateral Donors and Aid Conditionality in Post-Conflict Peacebuilding: The Case of Mozambique », Fournal of Modern African Studies, 48 (1), 2010, p. 143-169.
} 
domaine de la paix et de la justice, etc.). Cette élision est d'autant plus problématique que ce sont les civils qui accomplissent l'essentiel du travail d'établissement, de maintien et de consolidation de la paix. Deuxièmement, en se concentrant sur des groupes spécifiques de pacificateurs (soldats de la paix ou humanitaires), la plupart des études ont tendance à envisager leurs actions isolément, et à ignorer leurs interactions avec d'autres interventions concomitantes (établissement, maintien, consolidation de la paix, etc.). Ce réductionnisme conduit les analystes à attribuer l'amélioration ou, a contrario, la détérioration des situations conflictuelles à une seule catégorie d'organisations, alors que ces évolutions résultent souvent de l'œuvre combinée d'une variété d'acteurs locaux et extérieurs. Un tel biais nuit à l'intelligence des situations. Notre connaissance des modes d'action et des interactions entre intervenants demeure limitée, par-delà les rapports entre personnels militaires et humanitaires. En outre, il convient de dépasser la dichotomie entre acteurs locaux et extérieurs si l'on souhaite mieux apprécier les incidences matérielles et normatives des interventions de paix ${ }^{38}$.

Le fait de privilégier un seul type d'acteurs conduit à exclure du champ de la recherche une autre série de questionnements. Alors que les chercheurs adoptant une approche « par le haut » mettent en avant les différences et les similitudes éventuelles entre intervenants, les études « par le bas » prennent presque exclusivement en compte les différences et n'envisagent l'existence de possibles similitudes que pour dénoncer la vision occidentale et libérale de la paix que partageraient la plupart des protagonistes des missions de pacification. Une investigation plus systématique de ces caractéristiques communes, en particulier de celles forgées par des conceptions collectives qui ne s'inscrivent pas dans le paradigme de la paix libérale, fournirait pourtant des éclairages précieux.

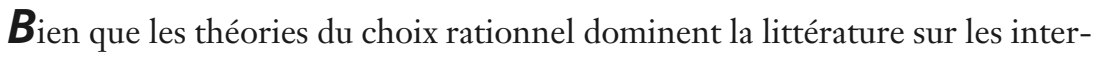
ventions de paix internationales, l'on dispose désormais d'un corpus de recherches substantiel qui démontre le rôle des multiples conceptions collectives dans le façonnage des opérations de paix. Celles-ci comprennent une culture mondiale principalement occidentale et libérale, des cultures régionales, nationales et locales, les cadres internes d'organisations internationales telles que la Banque mondiale ou les missions de maintien de la paix, ainsi que les regards professionnels propres à certains groupes (comme les négociateurs internationaux ou les acteurs humanitaires). Les principaux points de

38. D. Ambrosetti, «Urgences et normalités de gestionnaires face aux violences “des autres”. L'ONU et le Soudan », art. cité. 
débat tournent autour de deux questions : premièrement, parmi ces divers cadres interprétatifs, lequel prédomine et influence le plus profondément les interventions? Deuxièmement, quelle optique explique le mieux les pratiques d'intervention internationale, l'hypothèse de similitudes ou de différences entre intervenants?

Les approches «par le haut » ont largement contribué à notre compréhension des opérations de paix et de leur issue, mais leur propension à ignorer l'opération concrète des missions internationales sur le terrain paraît problématique. Faut-il le rappeler ? Le niveau microsocial ne peut être considéré comme une simple réplique du niveau macrosocial. Les approches «par le bas » permettent de corriger cette lacune. Néanmoins, en se limitant à l'étude des soldats de la paix, elles négligent trois domaines de recherche essentiels : l'influence des cadres d'intelligibilité sur les personnels non militaires, les interactions entre acteurs et fonctions dans les interventions de paix et la possible existence de similitudes entre intervenants.

Les deux approches ont par ailleurs une faiblesse commune : la plupart des auteurs choisissant de travailler sur des situations dans lesquelles les interventions ont échoué, nous disposons de peu d'informations sur la manière dont les cadres d'interprétation collectifs pourraient favoriser le succès des interventions. Il pourrait dès lors être heuristique d'étendre nos investigations à d'autres configurations. Les interventions de paix sont confrontées à des défis d'une telle envergure que la question la plus troublante n'est pas de savoir pourquoi certaines tentatives d'instauration de la paix échouent, mais plutôt pourquoi, parfois, certaines d'entre elles sont couronnées de succès ${ }^{39}$.

Traduit de l'anglais par Nadège Ragaru

L'auteur souhaite exprimer sa profonde gratitude à la Fondation Harry Frank Guggenheim et au United States Institute of Peace qui ont rendu son projet de recherche possible, à Sarah Marion Shore et à Anneke Dunbar-Gronke pour leur assistance de recherche, à Nathalie Duclos, Elisabeth King, Nadège Ragaru et aux deux relecteurs anonymes de Critique internationale pour leurs suggestions sur des versions antérieures de cet article. L'auteur reste seule responsable des éventuelles erreurs subsistant dans ce texte.

39. V. P. Fortna, Does Peacekeeping Work? Shaping Belligerents' Choices after Civil War, op. cit. ; L. M. Howard, UN Peacekeeping in Civil Wars, op. cit.. 
Séverine Autesserre est maître de conférences en science politique à la faculté Barnard de I'Université de Columbia (New York, USA). Elle travaille sur les guerres civiles, les interventions internationales, la construction de la paix et les politiques africaines. Son projet de recherche actuel étudie les influences culturelles sur les interventions de paix, notamment en République démocratique du Congo, au Soudan et au Burundi. Elle a publié The Trouble with the Congo: Local Violence and the Failure of International Peacebuilding (Cambridge University Press, 2010), "Hobbes and the Congo: Frames, Local Violence, and International Intervention », International Organization (63, 2009, p. 249-280) et "The Trouble with Congo - How Local Disputes Fuel Regional Violence ", Foreign Affairs (87 (3), 2008, p. 94-110). Adresse électronique : sa435@columbia.edu 